\title{
HEPATIC NECROSIS IN DISSEMINATED HERPES SIMPLEX*
}

\author{
BY \\ R. C. B. PUGH, G. H. NEWNS and J. A. DUDGEON \\ From The Hospital for Sick Children, Great Ormond Street, London
}

(RECEIVED FOR PUBLICATION OCTOBER 21, 1953)

Infection with herpes simplex virus is extremely common and the primary infection is frequently asymptomatic, as is shown by the high proportion of the adult population who possess herpes antibody but yet give no history of the clinical disease. In the symptomatic form, characterized by the appearance of a vesicular eruption on the skin or mucous membranes, considerable variation is seen both in the site and extent of the eruption, as well as in the severity of the accompanying constitutional reaction. Typically the infection is self-limiting and, even in the more extensive forms, recovery is usually complete in two or three weeks with the development of active immunity.

In generalized herpes simplex secondary lesions appear on the skin or mucosal surfaces as the result of dissemination of virus from the initial site of infection. In addition to direct spread on the surface which accounts for some of these lesions, particularly in eczematous patients, there is potent clinical evidence to support the view of spread by the blood stream. Laboratory evidence of viraemia, on the other hand, is fragmentary as the virus has only rarely been recovered from the blood stream (Ruchman and Dodd, 1950; Dudgeon, 1952; McNair Scott, Coriell, Blank and Burgoon, 1952).

There seems little doubt, however, that haematogenous spread is primarily responsible for an atypical form of disseminated herpes that has recently been reported from the United States by Zuelzer and Stulberg (1952). Their eight fatal cases fall into two groups; five newborn infants who were found to have widespread herpetic lesions in the viscera, and three older infants with less extensive visceral involvement. In both groups focal necrotic lesions were a prominent feature in the affected viscera and were invariably found in the liver. Fssentially similar hepatic lesions in premature infants were described by Hass (1935), who suggested

* This case was reported to the Pathological Society of Great Britain and Ireland on January 3, 1953. on morphological grounds that herpes virus was the causative agent, and by Quilligan and Wilson (1951), who recovered herpes virus from the liver in their case.

The case reported here is of an infant who died at the age of 6 weeks after a protracted, though initially mild, illness. At necropsy there was extensive focal necrosis of the liver from which herpes simplex virus was recovered.

\section{Case Report}

C.B. was the second child of Italian parents, and was born one week prematurely after a pregnancy that had been complicated by mild maternal toxaemia. Delivery was normal and her birth weight was $6 \mathrm{lb} .13 \mathrm{oz}$. The immediate post-natal period was uneventful except that a number of small 'spots' were found on the skin on the fourth day of life; they were not vesicular and disappeared within 24 hours.

At the age of 7 days soreness of the tongue and inside of the mouth was noted and this progressed to such an extent that breast feeding had to be discontinued on the twenty-first day. On the twenty-sixth day choking attacks with slight cyanosis occurred after each feed and the family doctor, noting some white patches in the mouth, diagnosed thrush. The child was first seen in the Casualty Department of The Hospital for Sick Children at the age of 27 days; she had a hoarse cry, the tongue was reddened, especially at the tip, but there was no evidence of thrush or ulceration of the tongue or buccal mucosa. She was thought to have had a recent stomatitis and a congenital deformity of the larynx was suspected. Five days later the infant was seen in the Out-patient Department because of a cough which had developed soon after her first attendance at the hospital; the choking attacks had ceased and feeds had been taken well, although she vomited small quantities of clear fluid between feeds. The baby had lost $1 \mathrm{oz}$. in weight and looked slightly dehydrated; there was a hoarse cry with marked inspiratory stridor and the tongue was still reddened but there was no evidence of thrush or ulceration of the buccal mucous membrane. She was admitted to hospital on the thirty-second day of life.

On examination after admission the infant was pale and a large quantity of mucus continually dribbled from 
the mouth. Her temperature was $99^{\circ} \mathrm{F}$., pulse 126 per minute, weight $7 \mathrm{lb}$. $13 \mathrm{oz}$. The tongue and buccal mucosa were uniformly reddened and there were a few small white patches on the palate. Streptococcus viridans and coagulase-positive staphylococci were cultured from the mouth but no monilia were isolated.

Local treatment with gentian violet and merthiolate produced an improvement in the oral lesions; the white patches disappeared but the buccal mucosa remained reddened. Feeding was difficult and on several occasions tube feeding was necessary. The weight remained stationary, but for the first eight days after admission there appeared to be no cause for anxiety. On the ninth day her condition suddenly worsened and the temperature rose to $100 \cdot 4^{\circ} \mathrm{F}$. She became cyanosed and numerous haemorrhagic lesions, some of which developed into abscesses from which $P$ s. pyocyanea was isolated, appeared on the limbs. Signs of a left-sided pneumonia developed. Systemic treatment with penicillin, streptomycin and chloromycetin was begun immediately, but there was steady deterioration in her condition and she died two days later, at the age of 42 days.

The mother and father were both healthy and gave no history of herpetic infection; there was no evidence that the mother had been exposed to herpes virus during her confinement.

An elder sibling died in this hospital in 1950 at the age of 3 months; the post-mortem diagnosis was interstitial pneumonitis, lung abscess and bronchiectasis following unresolved neonatal pneumonia. All the available histological material has been re-examined but there is no evidence that herpes virus was responsible for any of the changes found.

\section{Post-Mortem Appearances}

The necropsy was performed 44 hours after death. The body was that of a female infant in moderately good nutritional condition with pitting oedema of both legs below the knee (height $21 \frac{1}{2}$ in., body weight, $7 \mathrm{lb}$.). There were many rounded or irregularly shaped macular lesions on the skin of the legs, forearms, hands and left cheek; the majority were small $(0 \cdot 2-0 \cdot 3 \mathrm{~cm}$. in diameter) and were reddish-purple or brown, but several measured up to $1.5 \mathrm{~cm}$. in diameter and had a yellowish central zone which was surrounded by a reddish-purple halo.

The mucous membrane of the tongue, uvula and pharynx was uniformly smooth, pale and intact; the submucosa of the uvula and pharynx was thickened and oedematous. The oesophageal mucosa was diffusely congested but appeared intact. The liver weighed $200 \mathrm{~g}$. and the capsular surface was studded with numerous yellowish nodules of varying size (Fig. 1); the smaller ones were rounded, $0 \cdot 2$ or $0.3 \mathrm{~cm}$. in diameter, slightly raised above the surface and were often surrounded by a narrow zone of intense congestion. The larger lesions were up to $1.0 \mathrm{~cm}$. in diameter, and many had an irregular

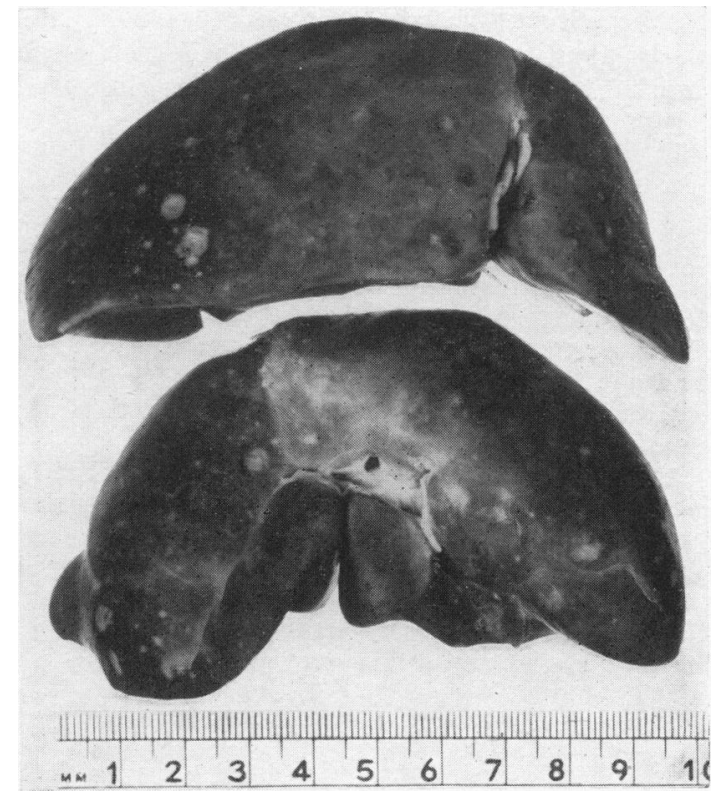

FIG. 1.-External surface of the liver: the necrotic lesions are widely scattered throughout both lobes and vary considerably in size.

outline and were slightly depressed centrally. The diaphragm and omentum also contained rounded necrotic foci which were attached to the necrotic areas on the surface of the liver by fibrinous adhesions. On section the liver parenchyma was swollen and congested and both lobes contained many widely scattered yellow lesions, similar to those seen on the capsular surface. The heart was of normal size but the myocardium was paler than normal. The larynx was normal. The bronchi were diffusely congested and all lobes of both lungs showed numerous subpleural petechiae, while the parenchyma was oedematous and congested with scattered areas of collapse and early bronchopneumonic consolidation. The brain weighed $479 \mathrm{~g}$. and showed considerable parenchymal oedema. Bisection of one of the larger skin lesions revealed a central necrotic core in the dermis and subcutis with congestion of the overlying epidermis. No other abnormalities were found on naked-eye examination.

A heavy growth of Ps. pyocyanea was obtained from a specimen of blood taken one hour after death.

\section{Laboratory Investigations}

The outstanding feature at necropsy was the presence of the abscess-like areas in the liver. Although it was known that pyaemia was present, it was felt that a more detailed examination of the 
necrotic liver tissue might prove of value, particularly as it was noticed that the lesions were remarkably similar to those reported and illustrated by Zuelzer and Stulberg (1952).

\section{Materials and Methods}

The essential details of the methods used have been summarized by Dudgeon (1951); the general scheme adopted in this case was as follows:

MiCrosCOPY.-A series of smears on clean glass slides was made from a representative area of necrosis. The films were air dried and stained by Gram and Giemsa's methods.

CULTURE.-The liver tissue was cultured aerobically and anaerobically. A $20 \%$ suspension of necrotic tissue was prepared in $10 \%$ broth saline containing 100 units of chloramphenicol per $\mathrm{ml}$. The suspension was centrifuged at 3,000 r.p.m. for 30 minutes before inoculation.

SEROLOGY.-Blood was collected by cardiac puncture. The serum after separation was grossly haemolysed.

Results.-The results of each investigation are as follows:

MicrosCOPY.-The direct smears showed a relative absence of leucocytes and bacteria in the necrotic liver tissue which suggested that the lesions were not pyaemic in nature. This was substantiated to some extent by the finding of degenerative nuclear changes in the hepatic cells. No intranuclear inclusions were seen.

Culture.-Ps. pyocanea was recovered from the liver. The addition of chloramphenicol inhibited its growth in the inoculated animals. Semiconfluent local lesions developed on the chorio-allantois of chick embryos after 48 hours' incubation. Morphologically these were very small and were similar to those produced by herpes simplex virus on primary isolation. Evidence of infection was also established both in newborn and in adult mice whereas no such signs developed in the rabbit or guinea-pig.

SEROLOGY.-Very little serum was obtained at necropsy and, as this was completely anticomplementary, antibody was estimated by the neutralization test using the plaque-counting method on the chorioallantois (McNair Scott, 1948). For technical reasons it was not possible to test the child's serum in as much detail as had been hoped; neither was it possible to obtain serum for testing from the mother and child on the same day. It would, therefore be inadvisable to draw rigid conclusions from the results that were obtained.

In the case of the mother and several posi- tive control sera (human) complete neutralization (i.e. $90 \%-100 \%$ ) was obtained with the sera diluted 1 in 80 . Known negative sera (acute phase samples from cases of primary herpes simplex) showed no neutralizing activity at 1 in 4 . On the other hand, the serum from the child showed a $50 \%$ reduction in the plaque count at 1 in 4 , from which it was concluded that antibody was present in low titre.

Herpes simplex virus was identified as the causative agent by the demonstration of intranuclear changes with inclusions in the infected tissues and by the inhibition of these changes with specific herpes antiserum.

\section{Histology}

The tongue, uvula and posterior pharyngeal wall showed similar changes although they varied in degree in different sites. The mucosa was everywhere intact but varied in thickness; in some areas it was thinned and oedematous and the nuclei were swollen and often bizarre; elsewhere it was hyperplastic and contained many mitotic figures together with some multinucleate cells. The sub-epithelial tissues were oedematous, contained prominent capillary vessels, and were infiltrated diffusely with many lymphocytes and some macrophages, polymorphs and eosinophils. The changes were non-specific and no inclusions were seen (Fig. 2).

In the upper oesophagus there were several sharply demarcated microscopic foci of recent coagulative

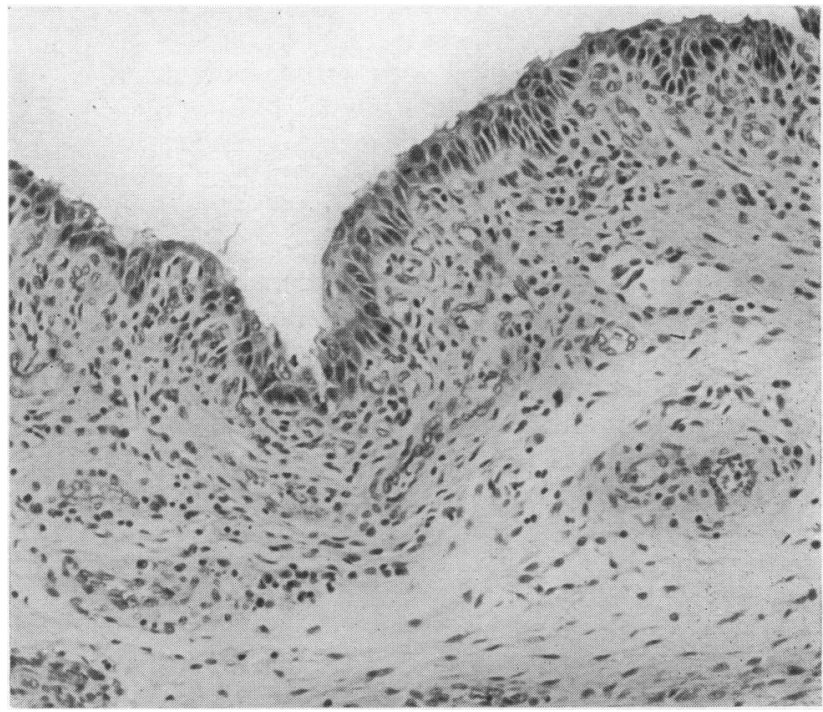

FIG. 2.-Posterior pharyngeal wall: the epithelium is ragged and the subepithelial tissues are well vascularized and contain chronic inflammatory cells. Haematoxylin and eosin $\times 140$. 


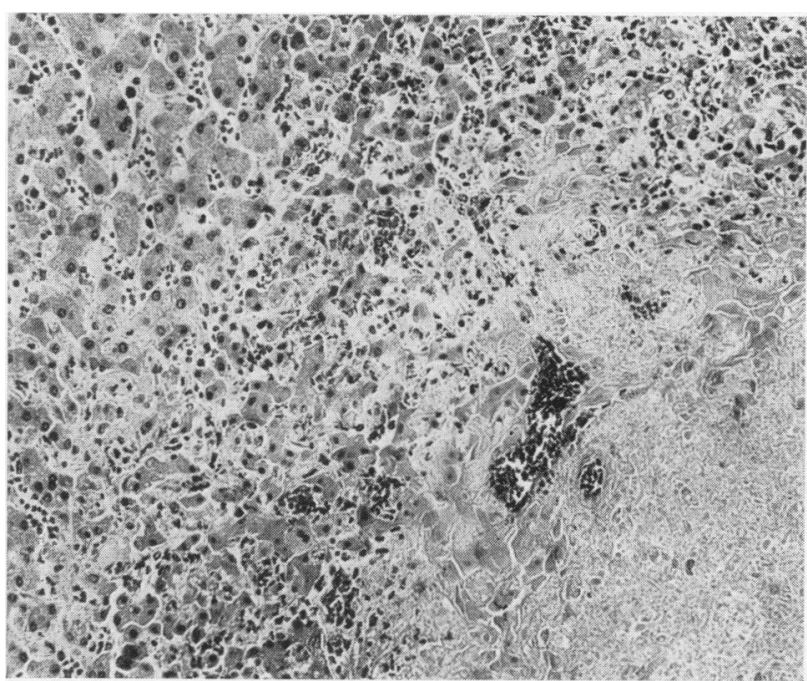

FIG. 3.-Liver: edge of a necrotic focus showing the clear line of demarcation between the necrotic and intact cells. No inflammatory cells are present in the necrotic tissue. Haematoxylin and eosin $\times 140$.

necrosis of the mucosa which were separated by intact but oedematous epithelium. The submucosa contained some lymphocytes, polymorphs and eosinophils. The changes were non-specific and were almost certainly the result of tube feeding. The lower oesophagus was normal.

The liver parenchyma contained many sharply demarcated areas of coagulative necrosis scattered irregularly throughout both lobes, often in close relation to blood vessels (Fig. 3). They varied in size from minute areas involving a few cells to extensive zones affecting one or more lobules. Many of the smaller lesions were of recent origin and, although nuclear and cytoplasmic degenerative changes were marked, the general architecture of the lobules was preserved. The larger lesions were older and the liver cells had been transformed into a structureless, eosinophilic mass with some distortion of the reticulin framework of the lobules. None of the lesions showed any evidence of healing. Many of the larger foci contained numerous Gram-negative bacilli. A noticeable feature was the relative absence of inflammatory exudate in the lesions, although at the periphery of some of the foci the sinusoids frequently contained large numbers of red cells together with some lymphocytes and macrophages. Even more striking, however, was the presence of nuclear changes in the parenchymal cells at the margins of the necrotic areas. Many of the nuclei were swollen and contained large nucleoli but there was no obvious alteration in the arrangement of the nuclear chromatin; these changes are quite commonly seen in herpes but are not specific for that disease. Other nuclei were considerably swollen and showed the features that are characteristic of a relatively early stage in the development of a herpes inclusion; the chromatin was marginated and nodular, while the central part of the nucleus was occupied by a homogeneous, deeply basophilic inclusion. Many cells contained the eosinophilic inclusions which are typical of a later stage in development (Cowdry type A inclusions); the nuclei were enlarged, the inclusions lay centrally and were surrounded by a clear 'halo', from which the nuclear chromatin had disappeared, while the nuclear membrane was deeply basophilic, thickened, nodular and sometimes crenated (Fig. 4). An occasional cell was seen in which the inclusion had evolved still further as the nucleus was now somewhat smaller and the nuclear membrane had contracted down on the eosinophilic inclusion so that the clear halo had disappeared.

The diaphragm and omentum contained focal necrotic areas that were essentially similar to those seen in the liver.

The remaining viscera, and especially the adrenals, the lungs and central nervous system, were examined

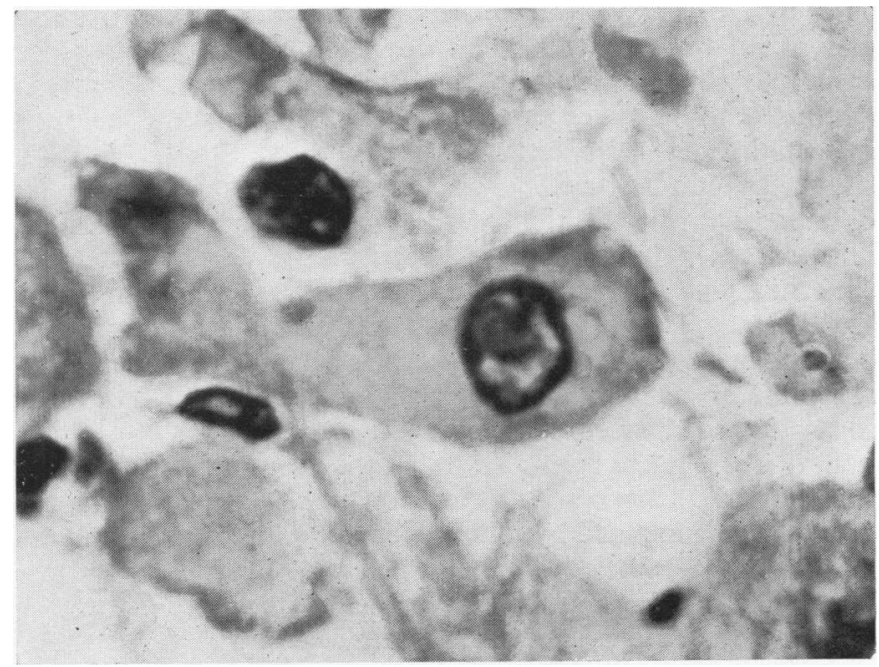

FIG. 4.-Liver: fully developed acidophilic intranuclear inclusion in a cell at the margin of a necrotic focus. Haematoxylin and eosin $\times 1,900$. 
for the presence of necrotic foci and intranuclear inclusions but no specific herpetic lesions were seen.

Section through one of the skin lesions showed that there was widespread necrosis of the dermis and subcutis, with partial and irregular necrosis of the epidermis. The necrotic tissues contained many Gram-negative bacilli but there was no inflammatory cellular reaction. In the absence of any specific nuclear changes, the most likely explanation is that these lesions were of pyaemic rather than viral origin.

The myocardium showed cloudy swelling and oedema. The presence of bronchopneumonia in the lungs was confirmed. The brain was congested and oedematous.

\section{Discussion}

At necropsy this child was found to have necrotic lesions in the liver and diaphragm; they were of distinctive appearance and were due to the local action of herpes simplex virus, which was recovered from the liver. There were healing inflammatory lesions in the oro-pharyngeal mucosa which could not be attributed definitely to the virus. Traumatic lesions, probably the result of repeated intubation, were seen in the upper oesophagus and there was also a terminal Ps. pyocyanea septicaemia with pyaemic skin lesions.

Visceral necrosis due to dissemination and focal multiplication of virus is a rare complication of certain virus diseases, such as vaccinia and varicella, but its occurrence in herpes simplex infection is only of recent recognition. Dissemination occurs by means of the blood stream, in much the same way as a pyaemia may occur in a bacterial infection, and like a pyaemia the extent of visceral involvement may vary from case to case although the liver, adrenals and lungs are particularly liable to contain metastatic viral lesions. A histological point that should be emphasized is that, apart from specific differences in the position of the inclusions within infected cells, the individual lesions in the viscera are strikingly similar in all three virus diseases. Also the microscopical appearances, especially the sharply outlined areas of parenchymal necrosis with minimal inflammatory reaction, are sufficiently characteristic to suggest that they are of viral origin.

In this case it has been shown that the liver lesions were produced by herpes virus and their presence can only be explained by blood stream spread from a focus of infection elsewhere in the body. There is no history of exposure to herpes virus nor is there unequivocal evidence as to the site of the primary lesion from which dissemination occurred. The skin 'spots' were transient and were not characteristic of herpes so that it seems likely that the portal of entry of virus was somewhere in the oro-pharynx. The clinical evidence does not exclude this and the histological changes are quite compatible with the healing phase of a primary herpetic lesion.

It is not known whether viraemia is a constant feature of herpes infections or whether it only occurs under certain abnormal circumstances, when it is responsible for the complications of the disease. More work needs to be done on this point but the fact that isolation of herpes virus from the blood stream has only been achieved on a few occasions indicates that the viraemia is almost certainly a transient one occurring early in the infection, as has been proved to be the case in the acute exanthems, such as smallpox (Downie, McCarthy and MacDonald, 1950; McCallum, McPherson and Johnstone, 1950).

When compared with the cases of disseminated herpes simplex reported by Zuelzer and Stulberg this case shows a number of interesting points of difference both in the course that the disease took and also in the extent of visceral involvement found at necropsy. Their two groups have already been referred to, Group 1 consisting of five newborn premature infants who died after a fulminating illness and had widespread visceral lesions due to herpes virus, and Group 2 comprising three older children with a gingivostomatitis and less extensive visceral involvement, largely confined to the liver. They attributed the different response to the virus in their two groups to the age at which infection occurred and suggested that the greater clinical severity and wider dissemination of virus in their Group 1 cases was due in part to the greater susceptibility of embryonic tissue. That age alone does not determine the clinical course and amount of visceral involvement is, we think, shown by the pattern of visceral lesions in our case, which resembles that of their Group 2 rather than their Group 1.

Although primary herpetic infection may occur at any age, the majority of individuals become infected during childhood, usually between the ages of 1 and 5 years (McNair Scott $e$ t al., 1952). Infection early in infancy, particularly in the neonatal period is, to our knowledge, extremely uncommon. This is probably due, in part at least, to protection afforded by maternal antibody which normally persists in the infant's circulation for the first four to six months of life (Anderson and Hamilton, 1949). The mother of our case was found to have herpes antibody in high titre four months after the birth of her child, and it is safe to assume, considering her age and the absence of any history of primary infection, that antibody was present at the time of her confinement. 
In which case, assuming that antibody passed across the placenta in the normal fashion, the child was infected whilst possessing maternal antibody. Similar serological findings were reported by Zuelzer and Stulberg (Case 1: Zuelzer and Stulberg, 1952).

Taking all factors into consideration it is difficult to offer an explanation of the finding of herpes antibody in such low titre in this infant as, under normal conditions, one would expect a close correlation between the antibody level in mother and child at the time of delivery. If we are in fact correct in our interpretation that antibody was present in low titre it would seem that this can be explained in one of two ways; either maternal antibody disappeared unduly rapidly or active immunity failed to develop pari passu with the infection. Unfortunately the serological investigations were too limited to allow any definite conclusions to be drawn on this point.

\section{Summary}

A fatal case of disseminated herpes simplex in a newborn infant is described. The oro-pharynx is suggested as the site of primary infection. Visceral lesions were confined to the liver and their herpetic aetiology was established by the pathological findings and isolation of virus. A marked difference was found in the serum antibody titre in mother and child.

We are grateful to Dr. Marjorie L. Pennill of the Mildmay Memorial Hospital, London, for details of the child's health during the first week of life. We are indebted to Dr. Martin Bodian for helpful criticism in the preparation of this paper and to Mr. Derek Martin for the illustrations.

\section{REFERENCES}

Anderson, S. G. and Hamilton, J. (1949). Med. J. Aust., 1, 308.

Downie, A. W., McCarthy, K. and MacDonald, A. (1950). Lancet, 2, 513.

Dudgeon, J. A. (1951), Gt Ormond Str. J., 1, 1.

(1952). A Communication to the Section of Pathology, the Royal Society of Medicine of London (March, 1952).

Hass, G. M. (1935). Amer. J. Path., 11, 127.

MacCallum, F. O., McPherson, C. A. and Johnstone, D. F. (1950) Lancet, 2, 514.

Quilligan, J. J. and Wilson, J. L. (1951). J. Lab. clin. Med., 38, 742.

Ruchman, I. and Dodd, K. (1950). Ibid., 35, 434.

Scott, T. F. McNair (1948). In Diagnostic Procedures for Virus and Rickettsial Diseases, p. 243. American Public Health Association, New York.

, Coriell, L., Blank, H. and Burgoon, C. F. (1952). J. Pediat., 41, 835 .

Zuelzer, W. W. and Stulberg, C. S. (1952). Amer. J. Dis. Child., 83,421

\section{Addendum}

Since this paper was written France and Wilmers have described herpes simplex hepatitis and encephalitis in newborn twins. (France, N. E. and Wilmers, M. J. (1953), Lancet, 1, 1181.) 